\title{
Study of Manufacturing and Measurement Reproducibility on a LaSer Textured Structured Surface
}

\author{
Gavin D. MacAulay ${ }^{1,2}$, Nicola Senin ${ }^{3}$, Claudiu L. Giusca ${ }^{1}$ and Richard K. Leach ${ }^{1}$ \\ ${ }^{1}$ Engineering Measurement Division, National Physical Laboratory, Teddington, United Kingdom, \\ TW110LW, gavin.macaulay@npl.co.uk \\ ${ }^{2}$ School of Engineering and Design, Brunel University, Uxbridge, United Kingdom \\ ${ }^{3}$ Department of Engineering, University of Perugia, Perugia, Italy
}

\begin{abstract}
In recent years there has been increasing interest in the use of structured surfaces to provide specific functional performance. Such surfaces often consist of localised micro-scale surface features with predetermined geometries. The performance of the feature manufacturing process affects the functional performance of the surface, and can be assessed by measurement of the resulting surface features. Measurement of the resulting micro-manufactured surface features necessitates use of areal optical surface topography instruments. However, conventional characterisation methods, based on areal surface texture parameters, often prove inadequate, and may fail to capture the relevant geometric properties needed for an effective dimensional verification. This paper investigates an alternative route to verification, based on the determination of geometric attributes of the micro-fabricated features. This approach allows for direct assessment of manufacturing process performance, by comparison of the geometric attributes with their nominal values. An example application is shown in which a micromachining process (laser texturing) is used to fabricate a periodic pattern of dimples, which provide a low friction bearing surface. In this paper, manufacturing process performance is assessed by characterisation of the diameter and out-of-roundness. Sources of uncertainty associated with these geometric parameters are also considered.
\end{abstract}

Keywords: Structured surfaces, Laser texturing, Surface metrology

\section{Introduction}

Structured surfaces are surfaces whose topography consists of deterministic features, designed to provide specific functional performance [1]. Structured surfaces are becoming increasingly popular due to their ability to provide improved functional performance for a number of applications, including: friction reduction, wettability and optical effects [2-7]. To manufacture such surfaces efficiently, fast, repeatable, low-cost micro-manufacturing techniques are required. Laser surface texturing (LST) is one such technique and is popular for the production of low-friction, structured surfaces [8-12].

Surface inspection and verification requires the capability to measure and analyse the geometries of the micro-fabricated features in comparison to their nominal counterparts. The ability to perform geometric assessment at the feature level can also serve as a tool to understand manufacturing process behaviour and performance, optimise process parameters, and compare manufacturing process variants. When manufacturing micro-scale features there is often a lack of geometrical specification and tolerances [13]. Additionally, there may be a poor understanding of how the surface geometry affects the functional performance. In such cases it is necessary for the geometry of the surface features to be well understood and highly repeatable.

The conventional surface metrology approach for analysing three-dimensional topography data is based on determining areal parameters (ISO 25178-2 [14]). However, the areal surface texture

\footnotetext{
${ }^{1}$ Current address: Faculty of Engineering, University of Nottingham, Nottingham NG7 2RD, UK
} 
parameters are statistical properties of the entire surface and so not ideally suited to characterising the geometric properties of individual surface features.

Recently, an alternative characterisation approach for structured surfaces has been proposed, in which individual surface features are identified and extracted as standalone units, so that they can be subjected to geometric verification $[6,12,15,16]$. When applied to micro-manufactured features of a structured surface, this approach enables the implementation of verification procedures similar to those used for quality inspection of standard-sized parts.

This work builds on previous research by the authors $[15,17]$ and considers the analysis of a test case consisting of cylindrical dimples in a periodic pattern, with the aim of producing a low friction structured surface for bearing applications. Several pattern designs with different nominal diameters are considered, while other geometric properties of the dimples are kept constant.

Dimple diameter and out-of-roundness are computed from measurements of a sample of the dimples in order to determine the reproducibility [18] of the manufacturing process, which could be considered as an indicator of process performance. Additionally, uncertainty in the measurements is considered. One component of the uncertainty is reproducibility of an individual measurement. This is considered separately from the component of reproducibility due to manufacturing. If reproducibility in the measurement is poor, good manufacturing quality may be masked by poor measurement data.

The diameter and out-of-roundness results for the test case are used to demonstrate how such a characterisation procedure, aimed at individual surface features, can be used to investigate manufacturing process behaviour and performance. This demonstration highlights the advantages and open issues of the approach.

Section 2 describes the samples and measurement approach used in this paper. Section 3 describes the analysis steps to determine dimple diameter and out-of-roundness, and how the data is considered. Section 4 presents the results of the initial measurements. Section 5 assesses the repeatability in measuring a single dimple. Section 6 considers effect of position in the field of view on the repeatability. Section 7 discusses the implications of these results as well as the strengths and limitations of the presented method.

\section{Specimens and sampling}

Three physical specimens with nominal diameters $50 \mu \mathrm{m}, 150 \mu \mathrm{m}$ and $300 \mu \mathrm{m}$ respectively were considered. Each specimen consisted of a silicon nitride disk with and a regular pattern of nominally cylindrical pits (dimples) designed for friction reduction. Dimples were manufactured via femtosecond-pulsed laser texturing with nominally $10 \mu \mathrm{m}$ depth and $20 \%$ coverage density. For each disk, a sample of 100 dimples, chosen at random from the several thousand on the surface, were measured using an Alicona InfiniteFocus G4 focus variation microscope. The microscope was setup with $20 \times$ objective lens, 0.40 numerical aperture, field of view $0.715 \mathrm{~mm} \times 0.544 \mathrm{~mm}$ and pixel size $0.438 \mu \mathrm{m} \times 0.438 \mu \mathrm{m}$. Using this configuration, the result of each measurement is a height-map containing one complete dimple topography. Figure 1 shows an example height-map (150 $\mu \mathrm{m}$ diameter dimple). The magnification was chosen as the highest magnification that would allow the largest dimples to fit into a single image.

In this paper, it was assumed that measurements of different dimples are independent and normally distributed. It is expected that there will be some spatial correlation between dimples on a disk. However, this correlation has been neglected due to the relatively small fraction of the population being sampled. Initial tests on the measurement data found that the results were approximately normally distributed. 


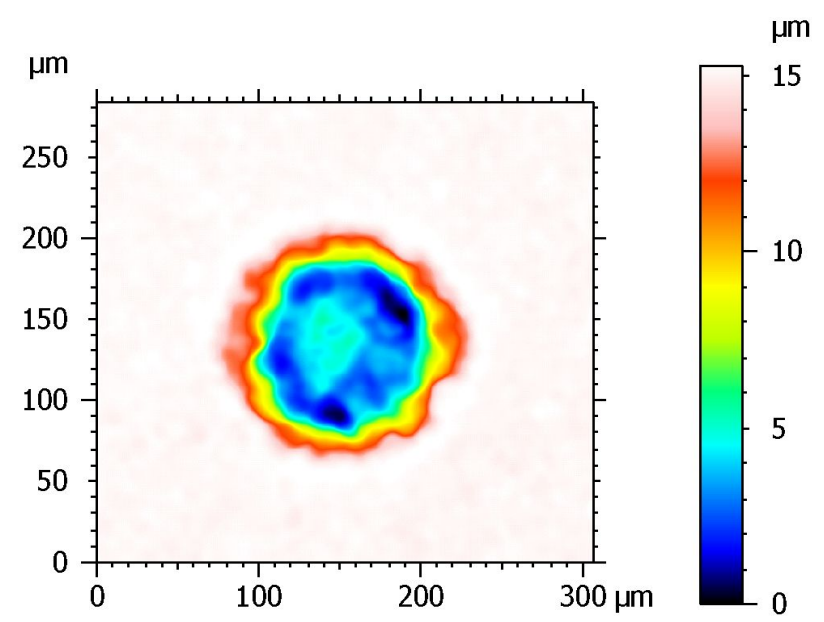

Figure 1: Height-map of a dimple with nominal diameter $150 \mu \mathrm{m}$. Image was cropped to exclude partial dimples appearing at the image boundaries.

\section{Computation and analysis of dimple diameter and out-of-roundness}

A dedicated procedure was developed to compute the dimple diameter and out-of-roundness from a measured topography. The procedure follows previous work on characterising structured surfaces in which individual features are identified and analysed geometrically. The general approach is illustrated in detail elsewhere $[12,15,17,19]$. The procedure consists of multiple steps, described below and summarised in figure 2 .

The procedure to identify and analyse measured dimples consists of five main steps. These are:

1. Pre-processing: The raw height map was filtered using a Gaussian kernel with $1.6 \mu \mathrm{m}$ standard deviation. The surface was then levelled by subtraction of a least-squares mean reference plane fitted to the background surface surrounding the dimples. This selective levelling ensures that different dimples are all referred to the same planar reference surface. Further details can be found in previous work [16].

2. Segmentation: A thresholding operation on the local image gradient was used to segment the surface. The local gradient was computed as the magnitude of the Sobel operator [20], as shown in figure 2a. A binary classification map was then obtained by applying a threshold at a gradient of 0.3 (figure $2 \mathrm{~b}$ ). Various other methods exist which could be used to perform a similar segmentation $[15,17]$. The importance of the choice of segmentation method and setting the correct threshold value is discussed further in section 7.

3. Post-processing and identification of the surface feature: The binary classification map produced by segmentation was further processed in order to better isolate the central dimple from its surroundings, as shown in figure 2c. The background surface should be flat compared to the dimples. Therefore, dimple regions were identified as high gradient areas, whereas low gradients were background regions. After this step, background regions, which are enclosed by dimple regions, were filled in and marked as part of the dimple. This filling accounts for misclassification of some dimple regions due to low local slope. Similarly, some background regions with high local slope may be misclassified as dimple regions. These regions were accounted for by reclassifying any small dimple regions (< 5000 pixels in area) as background. This reclassification should also account for incomplete dimples on the image boundary. The result after this step is a binary map with a single, connected region identifying the feature.

4. Determination of reference geometry: Since the nominal feature shape is cylindrical, a circular reference geometry was fitted to the boundary of the identified dimple. The resultant circle provides a 
suitable reference when determining the geometric properties of the feature. Figure $2 \mathrm{~d}$ shows the fitted circle superimposed onto the original topography.

5. Computation of the geometric properties: Dimple diameter error and out-of-roundness were calculated for each dimple. Dimple diameter was defined as the diameter of the fitted reference circle. The diameter error was calculated as the difference between the nominal and measured diameters. Using diameter error rather than measured diameter allows for comparison between dimples with different nominal diameter. The out-of-roundness was calculated as a peak to valley deviation of the dimple boundary from the fitted circle, i.e. the sum of the maximum positive and negative radial deviations from the fitted circle.

Due to the manufacturing process used, the magnitude of both the diameter error and out-of-roundness may be dependent on the nominal diameter. If this dependence is linear it can be accounted for by normalising the diameter error and out-of-roundness by the associated nominal diameter. This normalisation facilitates comparison of the significance of the geometric properties relative to the nominal diameter.

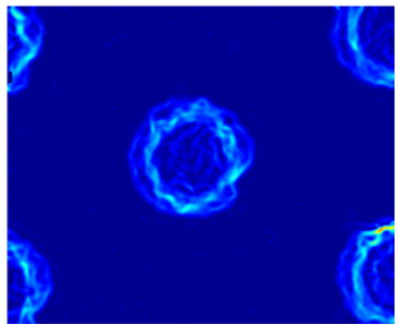

a)

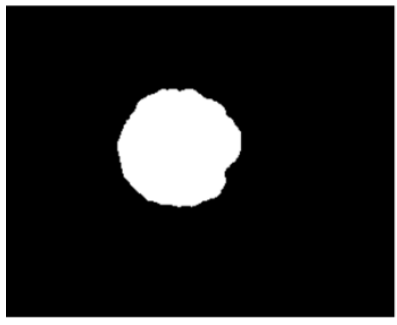

c)

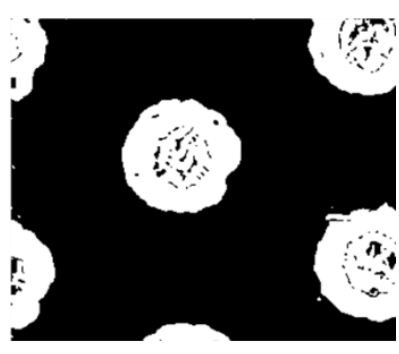

b)

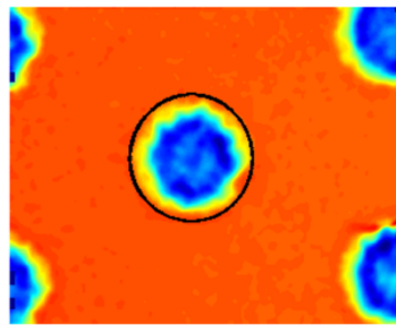

d)

Figure 2: The dedicated procedure for computing dimple diameter and out-of-roundness: a) gradient map calculated from the height data; b) segmentation map; c) post-processed map and feature identification; d) least-squares reference circle (black) fitted to the dimple boundary and superimposed onto the height-map.

\section{Assessment of measurement results}

The results of the measurements, described in section 3, are summarised in table 1 . The table contains absolute values (see figure 3 ) and values normalised by associated nominal diameter (figure 4).

Table 1: Mean and standard deviation (std) of diameter error and out-of-roundness for each set of samples. 


\begin{tabular}{lcccccc}
\hline & mean & std & mean & std & mean & std \\
\hline Diameter error/ $\mu \mathrm{m}$ & -4.64 & 3.38 & 5.79 & 1.69 & 1.67 & 4.19 \\
Normalised dia. error & -0.093 & 0.068 & 0.039 & 0.011 & 0.006 & 0.014 \\
Out-of-roundness/ $\mu \mathrm{m}$ & 8.63 & 3.18 & 15.36 & 2.59 & 18.29 & 5.45 \\
Normalised OoR & 0.17 & 0.064 & 0.10 & 0.017 & 0.061 & 0.018 \\
\hline
\end{tabular}

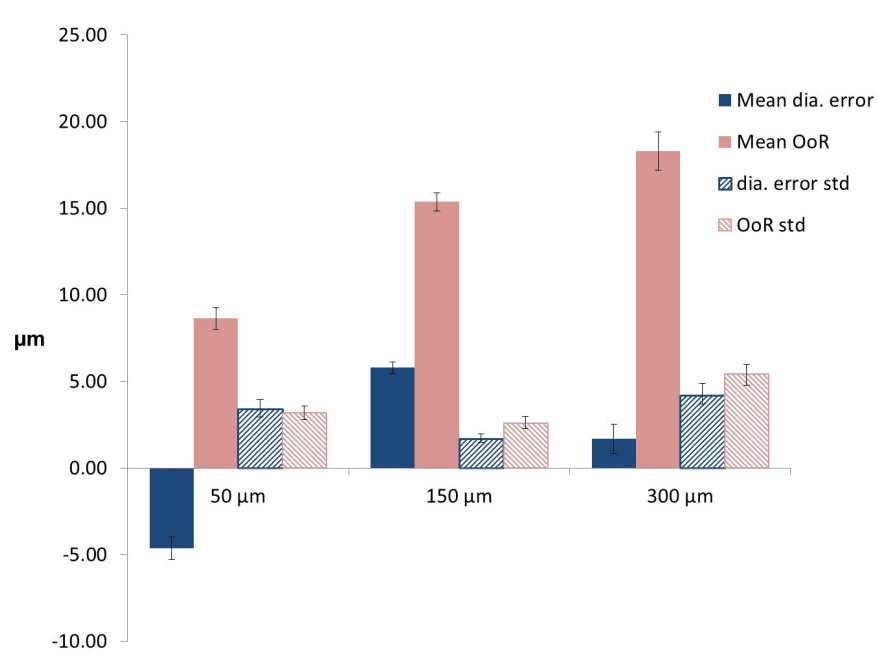

Figure 3: Means and standard deviation (std) values for diameter error and out-of-roundness (OoR) for each set of samples. Error bars indicate $95 \%$ confidence intervals, determined from repeatability of the measurements.

There appears to be no significant correlation between nominal diameter and either absolute or normalised mean diameter error. This lack of correlation may be the combined result of how the manufacturing process was optimised individually for each setup and the choice of threshold applied to the measurement data during the segmentation step. For the out-of-roundness there does appear to be some correlation with nominal diameter. From figure 4 the out-of-roundness becomes more significant relative to the nominal diameter as nominal diameter decreases. This relationship could be interpreted as a decrease in the quality of the edge as diameter decreases, which may be an important factor to consider if manufacturing smaller dimples.

The error bars in figures 3 and 4 do not account for type B measurement uncertainty components [21]. These components are assumed to be strongly correlated between measurements and were considered as an unknown bias in the results. Therefore, when comparing between measurements these components were ignored. It is assumed that any residual random component is small compared to the type A uncertainty components. However, if it was important to know the absolute value of the diameter error and out-of-roundness, then type B uncertainty components would have to be considered. An initial estimate of the magnitude of such components is given in section 7 . 


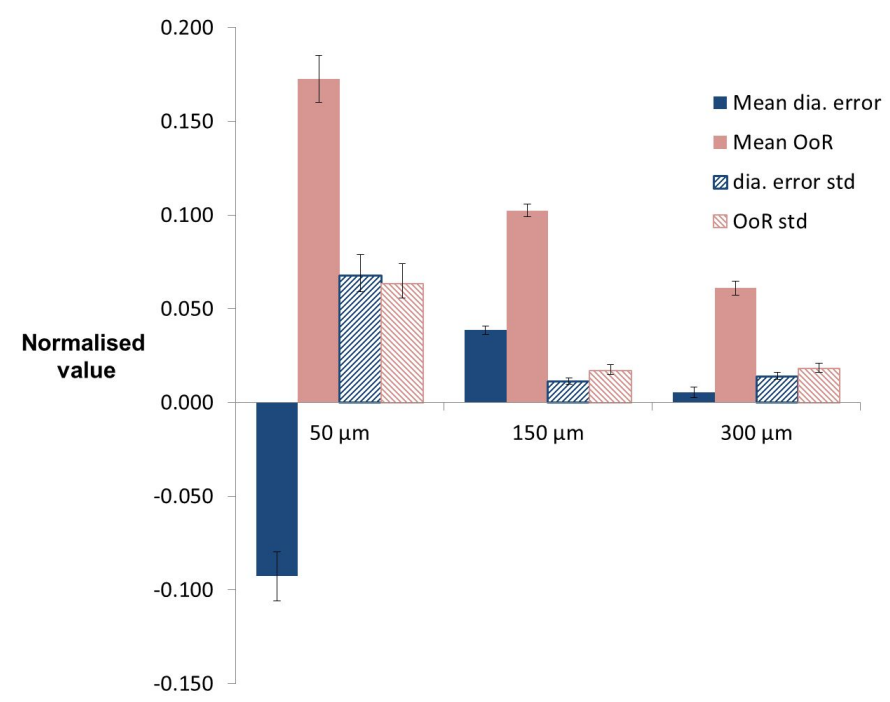

Figure 4: Means and standard deviation (std) values for diameter error and out-of-roundness (OoR) or each set of samples, after normalisation by the nominal diameter of the associated sample. Error bars indicate $95 \%$ confidence intervals, determined from repeatability of the measurements.

\section{Assessment of measurement reproducibility}

The requirement for measurement reproducibility to be significantly better than the manufacturing reproducibility was discussed in section 1 . One way to assess the measurement reproducibility is to measure the same dimple multiple times with the same measurement setup, with the position of the dimple in the field of view of the instrument varied for each measurement.

The measurement reproducibility was determined from measurements of three dimples on each disk. The use of multiple dimples per disk reduces the influence of variations in geometry and outliers on the determination of measurement reproducibility. Each dimple was measured at multiple positions within the field of view, defined by a $5 \times 5$ grid. The spacing of the grid varied with nominal dimple size to ensure the field of view was entirely covered as shown in figure 5 . At each position, ten repeat measurements were made, for a total of 250 measurements per dimple. The measured topography data was processed using the procedure described in section 3 , and the resulting diameters and out-of-roundness were used to determine the measurement reproducibility. 


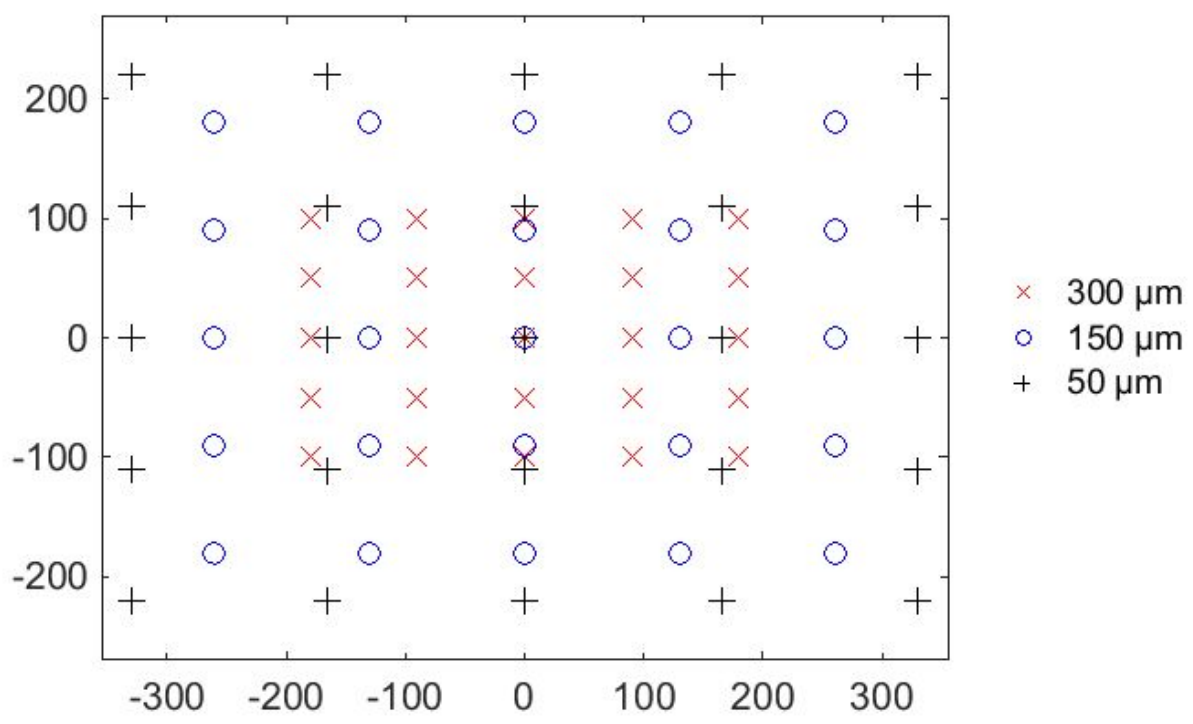

Figure 5: Grid of dimple centre positions for different nominal diameters in the field of view, in $\mu \mathrm{m}$. Axes cover the entire $715 \mu \mathrm{m} \times 544 \mu \mathrm{m}$ field of view of the instrument.

The measurement reproducibility is required to estimate the manufacturing reproducibility. The total reproducibility of geometric properties of measurements of random dimples can be approximated by the variance, $\sigma^{2}$, calculated as

$$
\sigma^{2}=\sigma_{m}^{2}+\sigma_{d}^{2}
$$

where $\sigma_{d}^{2}$ is the variance in their actual diameters, i.e. the manufacturing reproducibility, and $\sigma_{m}^{2}$ is the variance between difference measurements of the same dimple, i.e. the measurement reproducibility. The value of $\sigma^{2}$ can be calculated from the full set of measurement results and $\sigma_{m}^{2}$ can be calculated from the repeated measurements of a single dimple. Using these values, the relative importance of the manufacturing reproducibility, $\sigma_{d}^{2}$, can be assessed.

The reproducibility results on a single dimple, summarised in table 2 , show that the standard deviation of the diameter error is small compared to the standard deviation from measurements of multiple dimples and consistent across the three dimples considered. However, for the out-of-roundness, the standard deviation is much larger and has some extreme values, such as dimple 2 at $300 \mu \mathrm{m}$. This increased variability is to be expected, as the out-of-roundness is sensitive to extreme boundary positions. Therefore, small changes in the detected boundary could have a significant effect on the out-of-roundness, whereas the same changes would be insignificant when calculating the diameter through circle fitting.

Table 2: Summary of results of reproducibility tests showing diameter error and out-of-roundness (OoR).

\begin{tabular}{|c|c|c|c|c|c|c|c|c|c|}
\hline \multirow{2}{*}{ 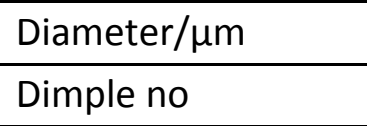 } & \multicolumn{3}{|c|}{50} & \multicolumn{3}{|c|}{150} & \multicolumn{3}{|c|}{300} \\
\hline & 1 & 2 & 3 & 1 & 2 & 3 & 1 & 2 & 3 \\
\hline 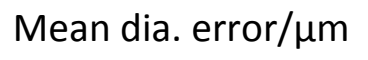 & -5.62 & -2.08 & -4.83 & 6.29 & 4.24 & 6.59 & 2.45 & 6.70 & -0.16 \\
\hline Std. dia. error/ $\mu \mathrm{m}$ & 0.17 & 0.16 & 0.14 & 0.14 & 0.14 & 0.11 & 0.083 & 0.090 & 0.082 \\
\hline Mean OoR/ $\mu \mathrm{m}$ & 6.97 & 8.92 & 7.67 & 18.70 & 11.40 & 13.51 & 14.52 & 10.32 & 8.49 \\
\hline Std. OoR/ $\mu \mathrm{m}$ & 0.25 & 0.25 & 0.44 & 0.68 & 0.23 & 0.39 & 0.53 & 1.23 & 0.25 \\
\hline
\end{tabular}


Based on (1), the importance of the measurement reproducibility of a single dimple can be considered as a fraction of the reproducibility when measuring multiple dimples, as shown in table 3 . The maximum variance from the three dimples in the reproducibility measurements has been used to avoid underestimating the importance of the measurement reproducibility. Measurement reproducibility was found to be approximately $5 \%$ of the reproducibility when measuring multiple dimples in the out-of-roundness and less than $1 \%$ in the diameter. Therefore, the measurement reproducibility is insignificant compared to other sources of uncertainty, such as the manufacturing reproducibility, and reproducibility in the measurement of multiple dimples is a good estimate of the manufacturing reproducibility.

Table 3: Ratios of the variance for diameter error and out-of-roundness (OoR) for the three dimple sizes. The ratio is calculated as the maximum variance due to measurement reproducibility on a single dimple as a percentage of the total variance when measuring multiple dimples.

\begin{tabular}{|c|c|c|c|}
\hline Diameter/ $\mu \mathrm{m}$ & 50 & 150 & 300 \\
\hline error & 0.26 & 0.71 & 0.046 \\
\hline OoR ratio & 1.96 & 6.83 & 5.12 \\
\hline
\end{tabular}

\section{Position dependence of measurement reproducibility}

In general, measurement data from optical instruments may contain position dependant distortions, such as the pin cushion effect [22]. Such distortions may contribute a significant position dependent term to the measurement reproducibility. This could be modelled by splitting the measurement reproducibility into position independent and position dependent components

$$
\sigma_{m}^{2}=\sigma_{r}^{2}+\sigma_{p}^{2}(x, y)
$$

where $\sigma_{m}^{2}$ estimates the total measurement reproducibility, $\sigma_{r}^{2}$, is the position independent component and $\sigma_{p}^{2}(x, y)$ is the position dependent component. The relative significance of the position dependant component can be determined from the reproducibility measurements on a single dimple.

For the diameter, position dependence can be visualised by plotting mean diameter error as a function of grid position, using bicubic interpolation between the grid points. This is shown in figure 6 for the three $300 \mu \mathrm{m}$ dimples. These results indicate that there is some correlated dependence on position across the three dimples. The residual differences between the position dependence map for each of the three dimples are to be expected due to the differences in: the actual geometry of the nominally identical dimples; the exact position of the dimples in the field of view; and the reproducibility of the measurement system.

A correction map can be produced by taking the average of these three maps, subtracting the mean of the full resultant average map to centre the map at zero, and normalising the map by the associated nominal diameter. The accuracy of the correction map is limited by the differences between the input maps. The correction maps for each dimple diameter are shown in figure 7. 


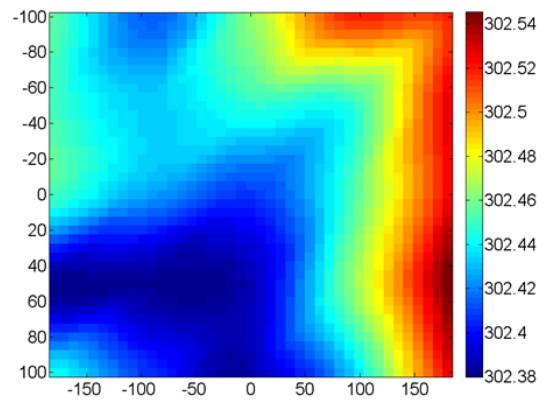

a)

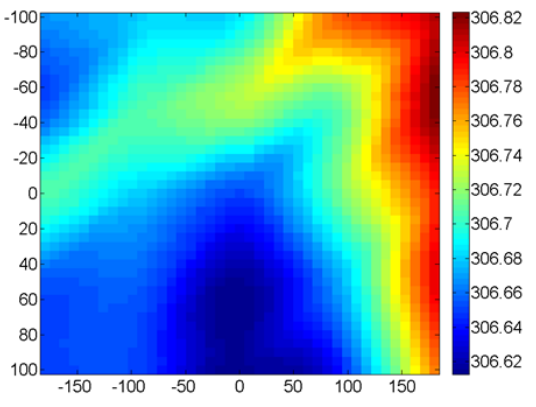

b)

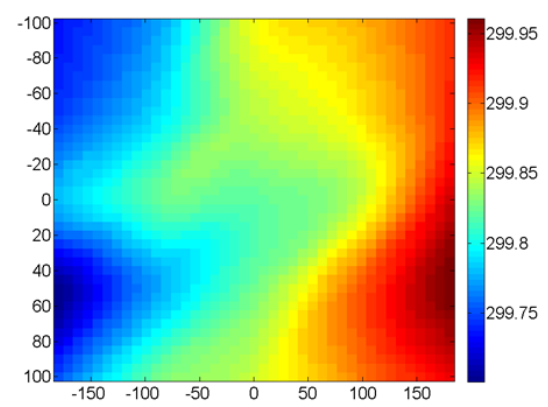

c)

Figure 6: Mean diameter at different centre positions (in micrometres), for the $300 \mu \mathrm{m}$ diameter disk reproducibility tests using bicubic interpolation. a), b) and c) show the three dimples measured.

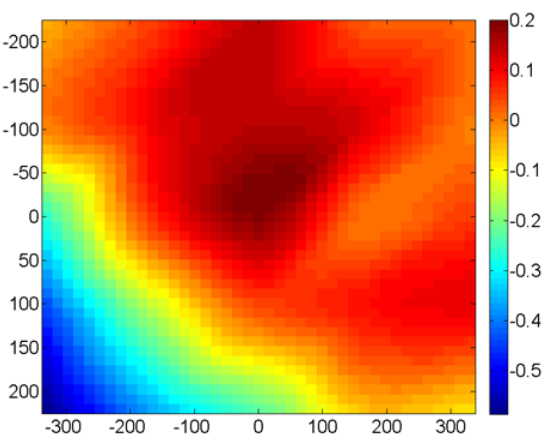

a)

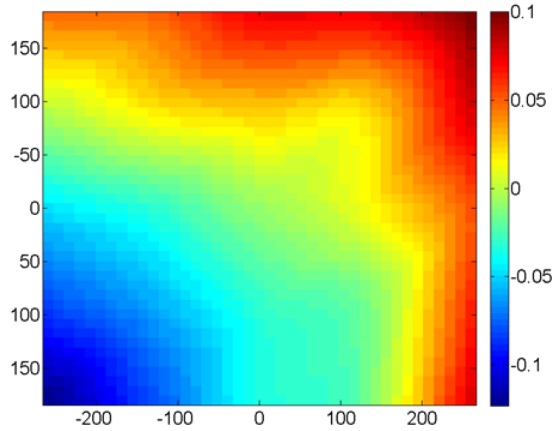

b)

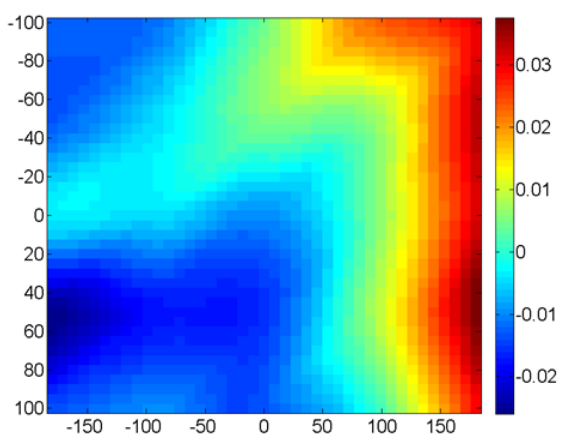

c)

Figure 7: Correction maps for diameter for the three disks. Dimple centre position (in micrometres) is plotted against correction factor. Correction factor is a percent of the nominal diameter. a), b) and c) show the 50, 150 and $300 \mu \mathrm{m}$ diameter disks respectively.

For these disks, the correction is very small; less than $1 \%$ in all cases. Therefore, it was deemed insignificant and not applied to the measurement data of multiple dimples, which has a far larger variance. For other situations, this may not be the case and it would be necessary to apply such a correction.

The dependence of out-of-roundness on position also was investigated. However, no correlation could be identified from the measurements (see figure 8 for data for the $300 \mu \mathrm{m}$ disk). The absence of correlation indicates that the position dependent variation in out-of-roundness appears to be insignificant relative to position independent variation in out-of-roundness. 


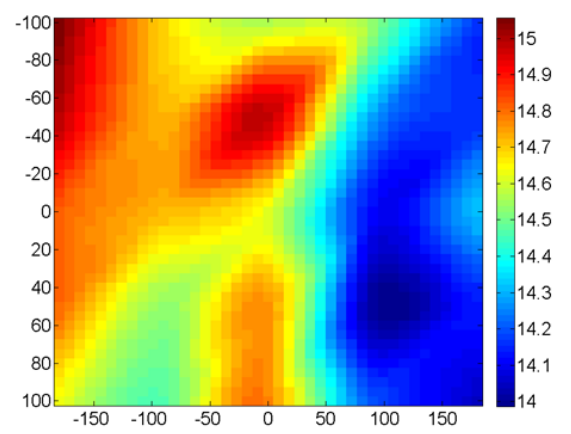

a)

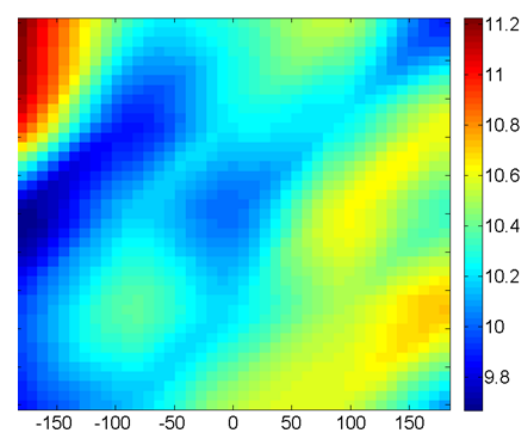

b)

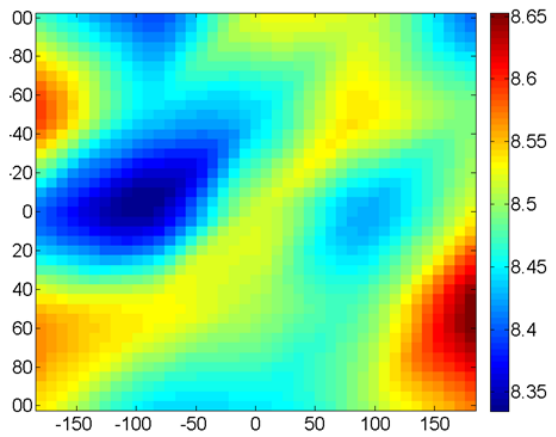

c)

Figure 8: Mean out-of-roundness at different centre positions (in micrometres), for the $300 \mu \mathrm{m}$ diameter disk reproducibility tests using bicubic interpolation. a), b) and c) show the three dimples measured.

\section{Discussion}

It is important to consider why deviations in the dimple boundary occur when the same feature is measured multiple times. Closer analysis shows that significant deviations can occur when there is a ridge on the boundary with a saddle close to the threshold value. When segmentation occurs as described in step 2 of section 3 noise in the measurement can cause the saddle to drop below the threshold creating a break in the boundary that will not be filled in during post processing described in section 3. This effect is demonstrated in figure 9. It is not straightforward to predict when such a situation will occur and it can cause significant changes in the results, particularly the out-of-roundness. These changes can lead to higher than expected standard deviation in some measurements such as in table 2 for $300 \mu \mathrm{m}$ dimple 2. This is a limitation in the characterisation method used and for other applications it may be of interest to investigate other segmentation methods, such as morphological segmentation [23,24] or active contours [25], which should be more stable in this respect. Alternatively additional post-processing steps could be added to mitigate the issue. 


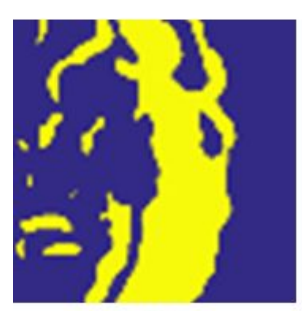

b)

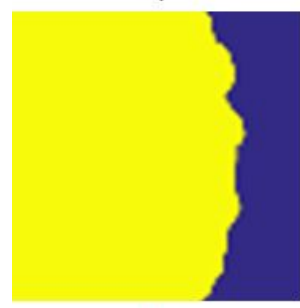

d)

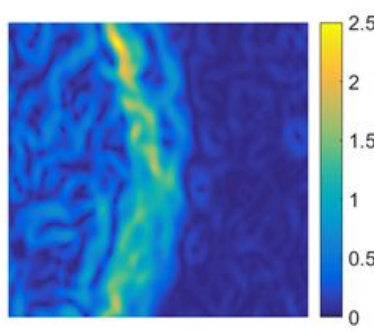

a)

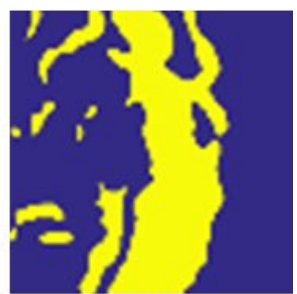

c)

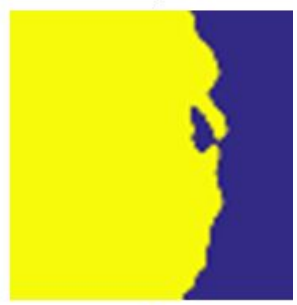

e)

Figure 9: Example of instability in thresholding algorithm. a) Section of gradient map of $300 \mu \mathrm{m}$ dimple. b) Binary map after segmentation (yellow shows regions marked as features). c) Segmentation of another measurement of the same feature. Note the break in the boundary in the centre right of the image. d) Image b) after post-processing. e) Image c) after post-processing. The gap in the boundary has not been filled in distorting the boundary.

In this paper it has been assumed that, because a random sample of dimples was selected, the measurements of different features were uncorrelated. However, there may be significant spatial correlation between features nearby on the surface. Such correlation is related to the manufacturing process and is outside the scope of this work. Further research is necessary to assess, quantify and account for any correlation and the effect it has on the statistics of the measured properties. For the results in this paper, samples were randomly selected from across the entire disk and are a very small fraction of the total population, so the effect of any correlation should be negligible for these measurements.

The methods presented here do not account for any Type B errors in the measurement. Such errors can be caused by many factors, such as amplification in the lateral or vertical scales. The focus variation instrument used in this paper is calibrated to an uncertainty in the lateral scales of $2 \%$. This corresponds to a type B uncertainty of $1 \mu \mathrm{m}, 3 \mu \mathrm{m}$ and $6 \mu \mathrm{m}$ in the diameter of the $50 \mu \mathrm{m}, 150 \mu \mathrm{m}$ and $300 \mu \mathrm{m}$ disks respectively. This lateral scales uncertainty term is a significant component to the uncertainty in the measurement of diameter error, and larger than the type A uncertainty in the mean reported in figure 3 . The lateral scales uncertainty term is a much less significant component of the uncertainty of the out-of-roundness measurement. Due to the comparative nature of the parameter the uncertainty will be $2 \%$ of the out-of-roundness value, which is small compared to other sources of uncertainty.

However, it can be assumed that the effect of lateral scales is constant between different measurements. Therefore, the lateral scale results in a constant scaling factor, which will cancel when comparing between measurements. If this were not the case then the position dependant repeatability would be much higher.

An additional source of uncertainty is the lateral resolution. For focus variation microscopes, the lateral resolution is determined by the software and for all experiments was set to $3 \mu \mathrm{m}$. One way to 
model the effect of the lateral resolution on the position of the feature boundary is by a triangular distribution with a base length of twice the lateral resolution. For these experiments, the standard deviation of this distribution is $1.2 \mu \mathrm{m}$. The primary effect of lateral resolution will be on the out-of-roundness, where it will affect both the minimum and maximum points. The combined uncertainty of these two points corresponds to an additional uncertainty in the out-of-roundness of 1.7 $\mu \mathrm{m}$. For the diameter, the effect of lateral resolution will be negligible, as the variations are assumed to average out around the circle. However, this is an overly simplified model; in practice the effect of lateral resolution is highly dependent on local geometry and must have significant spatial correlation, otherwise the detected boundaries would not appear smooth. It is reasonable to assume, due to the similar geometries between dimples, that the effect of the lateral resolution will be similar between different measurements. Therefore, when comparing between results lateral resolution terms will approximately cancel and can be neglected.

The effect of the analysis process on the measurement should also be considered. The dimple boundary is only defined by the method used in the analysis. If the analysis method is changed, either by using a different algorithm or by changing the threshold used, then the detected boundary and corresponding dimple properties will change. A deeper understanding of how these properties are defined in the manufacturing process is needed to determine the optimal way to define the boundary. For example, by changing the threshold a different boundary can be produced with different dimensional properties. However, this boundary may still reasonable, but different, results. Further research is needed understand how these choices should be made and how they effect the measurement results and associated uncertainties. In general, the choice of threshold should be strongly influenced by the intended function of the surface.

\section{Conclusions}

This paper has developed an approach to measure geometric properties of micro-scale features on structured surfaces and to consider the sources of uncertainty associated with such measurements. The manufacturing process performance of laser manufactured dimples of different nominal diameters was used as an example to demonstrate this approach. There was found to be a strong correlation between out-of-roundness and nominal diameter, although this was not the case for diameter error.

The measurement reproducibility was investigated by repeated measurement of a single dimple. It was found that the measurement reproducibility was small compared to the manufacturing reproducibility. Therefore, the reproducibility when measuring multiple dimples provides a good estimate of manufacturing reproducibility. A small amount of position dependence in the diameter was also detected. However, this was insignificant compared to other sources of uncertainty.

An initial consideration of type B uncertainties associated with such measurements was also given. However further work is necessary to give a better estimate of type B uncertainties and the correlation between uncertainties in different measurements.

\section{Acknowledgements}

This work was funded by the National Measurement System Innovation R\&D Programme 2012 to 2015.

\section{References}

[1] K.J. Stout, L. Blunt, A contribution to the debate on surface classifications-random, systematic, unstructured, structured and engineered, Int. J. Mach. Tools Manuf. 41 (2001) 2039-2044. doi:10.1016/S0890-6955(01)00069-4. 
[2] A.A.G. Bruzzone, H.L. Costa, P.M. Lonardo, D.A. Lucca, Advances in engineered surfaces for functional performance, CIRP Ann. - Manuf. Technol. 57 (2008) 750-769.

doi:10.1016/j.cirp.2008.09.003.

[3] C.J. Evans, J.B. Bryan, Structured, textured or engineered surfaces, CIRP Ann. - Manuf. Technol. 48 (1999) 541-556. doi:10.1016/S0007-8506(07)63233-8.

[4] C.W. Extrand, S.I. Moon, P. Hall, D. Schmidt, Superwetting of structured surfaces, Langmuir. 23 (2007) 8882-90. doi:10.1021/la700816n.

[5] E. Martines, K. Seunarine, H. Morgan, N. Gadegaard, C.D.W. Wilkinson, M.O. Riehle, Superhydrophobicity and superhydrophilicity of regular nanopatterns, Nano Lett. 5 (2005) 2097-103. doi:10.1021/nl051435t.

[6] L.B. Kong, C.F. Cheung, X.Q. Jiang, W.B. Lee, S. To, L. Blunt, et al., Characterization of surface generation of optical microstructures using a pattern and feature parametric analysis method, Precis. Eng. 34 (2010) 755-766. doi:10.1016/j.precisioneng.2010.04.005.

[7] T.R. Thomas, Roughness and function, Surf. Topogr. Metrol. Prop. 2 (2013) 014001. doi:10.1088/2051-672X/2/1/014001.

[8] A. Kovalchenko, O. Ajayi, A. Erdemir, G. Fenske, I. Etsion, The effect of laser surface texturing on transitions in lubrication regimes during unidirectional sliding contact, Tribol. Int. 38 (2005) 219-225. doi:10.1016/j.triboint.2004.08.004.

[9] G. Ryk, Y. Kligerman, I. Etsion, Experimental Investigation of Laser Surface Texturing for Reciprocationg Automotive Components, Tribol. Trans. 45 (2002) 444-449.

[10] I. Etsion, State of the Art in Laser Surface Texturing, J. Tribol. 127 (2005) 248-253. doi:10.1115/1.1828070.

[11] P. Andersson, J. Koskinen, S. Varjus, Y. Gerbig, H. Haefke, S. Georgiou, et al., Microlubrication effect by laser-textured steel surfaces, Wear. 262 (2007) 369-379. doi:10.1016/j.wear.2006.06.003.

[12] L. Blunt, S. Xiao, The use of surface segmentation methods to characterise laser zone surface structure on hard disc drives, Wear. 271 (2011) 604-609. doi:10.1016/j.wear.2010.06.014.

[13] H.N. Hansen, K. Carneiro, H. Haitjema, L. De Chiffre, Dimensional Micro and Nano Metrology, CIRP Ann. - Manuf. Technol. 55 (2006) 721-743. doi:10.1016/j.cirp.2006.10.005.

[14] ISO, ISO 25178-2 Geometrical Product Specifications (GPS) - Surface Texture: Areal - Part 2: Terms, definintions and surface texture parameters, 2011 (2012).

[15] N. Senin, G. MacAulay, C. Giusca, R.K. Leach, On the characterisation of periodic patterns in tessellated surfaces, Surf. Topogr. Metrol. Prop. 2 (2014) 025005. doi:10.1088/1468-6996/2/2/025005.

[16] N. Senin, L.A. Blunt, R.K. Leach, S. Pini, Morphologic segmentation algorithms for extracting individual surface features from areal surface topography maps, Surf. Topogr. Metrol. Prop. 1 (2013) 015005. doi:10.1088/2051-672X/1/1/015005.

[17] G.D. MacAulay, N. Senin, C.L. Giusca, R.K. Leach, Comparison of segmentation techniques to determine the geometric parameters of structured surfaces, Surf. Topogr. Metrol. Prop. 2 (2014) 044004. doi:10.1088/2051-672X/2/4/044004.

[18] JCGM, International vocabulary of metrology - Basic and general concepts and associated terms, (2012) http://www.bipm.org/utils/common/documents/jcgm/JCGM_200_2012.pdf. 
[19] N. Senin, L. Blunt, Characterization of individual areal features, in: R.K. Leach (Ed.), Characterisation Areal Surf. Texture, Springer, 2013: pp. 179-216.

[20] R.C. Gonzalez, R.E. Woods, Digital Image Processing, 3rd ed., Prentice Hall, 2008.

[21] JCGM, Guide to the expression of uncertainty in measurement, (2008) http://www.bipm.org/utils/common/documents/jcgm/JCGM_200_2008_E.pdf.

[22] A. Henning, C. Giusca, A. Forbes, I. Smith, R. Leach, J. Coupland, et al., Correction for lateral distortion in coherence scanning interferometry, CIRP Ann. - Manuf. Technol. 62 (2013) 547-550. doi:10.1016/j.cirp.2013.03.026.

[23] P.J. Scott, Pattern analysis and metrology: the extraction of stable features from observable measurements, Proc. R. Soc. A Math. Phys. Eng. Sci. 460 (2004) 2845-2864. doi:10.1098/rspa.2004.1291.

[24] A. Bleau, J.L. Leon, Watershed-Based Segmentation and Region Merging, Comput. Vis. Image Underst. 77 (2000) 317-370. doi:10.1006/cviu.1999.0822.

[25] T.F. Chan, L.A. Vese, Active contours without edges., IEEE Trans. Image Process. 10 (2001) 266-77. doi:10.1109/83.902291. 